\title{
Article \\ African Swine Fever Virus K205R Induces ER Stress and Consequently Activates Autophagy and the NF-KB Signaling Pathway
}

\author{
Qi Wang 1,2,3,+, Luyu Zhou 1,2,3,+, Jiang Wang 1,2,3,+, Dan Su 1,2,3, Dahua Li 1,2,3, Yongkun Du 1,4, \\ Guoyu Yang ${ }^{2,3,4,5}$, Gaiping Zhang ${ }^{1,4, *}$ and Beibei Chu ${ }^{1,2,3,4, * \mathbb{D}}$
}

check for updates

Citation: Wang, Q.; Zhou, L.; Wang, J.; Su, D.; Li, D.; Du, Y.; Yang, G.; Zhang, G.; Chu, B. African Swine Fever Virus K205R Induces ER Stress and Consequently Activates Autophagy and the NF- $\mathrm{KB}$ Signaling Pathway. Viruses 2022, 14, 394. https:/ / doi.org/10.3390/v14020394 Academic Editor: Chao-Nan Lin

Received: 20 January 2022

Accepted: 11 February 2022

Published: 15 February 2022

Publisher's Note: MDPI stays neutral with regard to jurisdictional claims in published maps and institutional affiliations.

Copyright: (C) 2022 by the authors. Licensee MDPI, Basel, Switzerland. This article is an open access article distributed under the terms and conditions of the Creative Commons Attribution (CC BY) license (https:// creativecommons.org/licenses/by/ $4.0 /)$.
1 College of Veterinary Medicine, Henan Agricultural University, Zhengzhou 450046, China; wq18839830417@sina.com (Q.W.); zhouluyu12@sina.com (L.Z.); wangjiang@henau.edu.cn (J.W.); sudan98@sina.com (D.S.); lidahua52@sina.com (D.L.); duyongkun@henau.edu.cn (Y.D.)

2 Key Laboratory of Animal Biochemistry and Nutrition, Ministry of Agriculture and Rural Affairs, Zhengzhou 450046, China; yangguoyu@henau.edu.cn

3 Key Laboratory of Animal Growth and Development, Henan Agricultural University, Zhengzhou 450046, China

4 International Joint Research Center of National Animal Immunology, Henan Agricultural University, Zhengzhou 450046, China

5 College of Animal Science \& Techmology, Henan University of Animal Husbandry and Economy, Zhengzhou 450047, China

* Correspondence: zhanggaiping2003@yahoo.com.cn (G.Z.); chubeibei@henau.edu.cn (B.C.)

$\dagger$ These authors contributed equally to this work.

\begin{abstract}
African swine fever virus (ASFV) is responsible for enormous economic losses in the global swine industry. The ASFV genome encodes approximate 160 proteins, most of whose functions remain largely unknown. In this study, we examined the roles of ASFV K205R in endoplasmic reticulum (ER) stress, autophagy, and inflammation. We observed that K205R was located in both the cytosolic and membrane fractions, and formed stress granules in cells. Furthermore, K205R triggered ER stress and activated the unfolded protein response through activating the transcription factor 6, ER to nucleus signaling 1, and eukaryotic translation initiation factor 2 alpha kinase 3 (EIF2AK3/PERK) signaling pathways. Moreover, K205R inhibited the serine/threonine kinase 1 and the mechanistic target of the rapamycin kinase signaling pathway, thereby activating unc-51 like autophagy activating kinase 1, and hence autophagy. In addition, K205R stimulated the translocation of P65 into the nucleus and the subsequent activation of the nuclear factor kappa B (NF- $\mathrm{B}$ ) signaling pathway. Inhibition of ER stress with a PERK inhibitor attenuated K205R-induced autophagy and NF- $\mathrm{BB}$ activation. Our data demonstrated a previously uncharacterized role of ASFV K205R in ER stress, autophagy, and the NF- $\mathrm{kB}$ signaling pathway.
\end{abstract}

Keywords: ASFV K205R; stress granules; ER stress; autophagy; NF-KB

\section{Introduction}

African swine fever virus (ASF), the only known DNA arbovirus, is prevalent in Africa, Europe, and Asia [1]. It causes a highly infectious and fatal hemorrhagic disease affecting the global swine industry [2]. Its genome contains approximately 160 major open reading frames (ORFs) and encoding products, including enzymes, structural proteins, and scaffolding proteins [3]. Some ASFV ORFs have been shown to be involved in regulating viral replication and host antiviral responses. For instance, ASFV A224L, A179L, EP153R, and DP71L inhibit apoptosis and prevent premature cell death, while ASFV E199L induces mitochondria-dependent apoptosis, supporting viral replication [4,5]. In addition, ASFV MGF-505-7R and MGF-505-11R negatively regulate the stimulator of IFN genes (STING)dependent antiviral responses [6,7]. However, the function of most ASFV ORFs remains largely unknown. Given the lack of an effective vaccine against ASFV, it would be of great 
significance to characterize the function of ASFV proteins, providing new insight into ASFV vaccine development.

Various viruses induce endoplasmic reticulum (ER) stress and the subsequent unfolded protein response (UPR) [8]. Three signaling pathways, involving eukaryotic translation initiation factor 2 alpha kinase 3 (EIF2AK3/PERK), ER to nucleus signaling 1 (ERN1/IRE1), and activating transcription factor 6 (ATF6), are essential for the UPR [9]. ASFV structural protein p17 induces the generation of ER stress-reactive oxygen species, which inhibit cell proliferation [10]. ASFV activates the ATF6 branch of UPR, thus promoting infection [11]. CCAAT-enhancer-binding protein homologous protein (CHOP) is induced by ATF6 [12], but ASFV does not activate $\mathrm{CHOP}$ and instead inhibits the induction of $\mathrm{CHOP}$ through several exogenous stimuli [13]. Whether there are other ASFV proteins modulating ER stress remains unknown.

Autophagy is a conserved self-degradative process, which is important for cellular homeostasis and host-pathogen interactions [14]. ASFV E199L promotes autophagy through interacting with pyrroline-5-carboxylate reductase 2 and down-regulates its expression [15]. ASFV MGF505-11R interacts with the stimulator of interferon response cGAMP interactor 1 and stimulates its degradation through autophagy, suggesting that ASFV evades the innate immune response through hijacking autophagy [7]. In contrast, ASFV A179L has been found to inhibit autophagosome formation through interaction with Beclin-1 [16].

ASFV K205R has been found to be expressed in the early stages of infection, from $4 \mathrm{~h}$ post-infection, and is located in viral factories. Therefore, the K205R gene has received extensive research attention [16]. Adenovirus-vectored ASFV K205R elicits robust immune responses in swine [17]. ASFV K205R has high antigenicity and can be recognized by hyperimmune antisera from infected pigs, suggesting that K205R has the potential to be used for the detection of ASFV-specific antibodies [18]. In the present study, we report that ASFV K205R activates autophagy and the inflammatory response through triggering ER stress. Our results indicated a novel function of ASFV K205R.

\section{Materials and Methods}

2.1. Cells

The 3D4/21 (ATCC, CRL-2843), PK-15 (ATCC, CCL-33), and HeLa (ATCC, CCL-2) cells were cultured in DMEM (Gibco, Waltham, MA, USA) supplemented with 10\% FBS (Gibco), 100 units $/ \mathrm{mL}$ penicillin, and $100 \mathrm{mg} / \mathrm{mL}$ streptomycin sulfate (Sangon, Shanghai, China). All cells were grown in monolayers at $37^{\circ} \mathrm{C}$ in $5 \% \mathrm{CO}_{2}$. The 3D4/21 P65 ${ }^{-/-}$, PK-15 ATG5 ${ }^{-/-}$, and Beclin-1-1- cells were used and cultivated as previously described $[19,20]$.

\subsection{Chemicals and Antibodies}

GSK2606414 (S7307) was ordered from Selleck (Pittsburgh, PA, USA), and LPS (HYD1056) was ordered from MedChemExpress (Monmouth Junction, NJ, USA). The antibodies, including anti-LC3 (12741), anti-LC3-II (3868), anti-P62 (5114), anti-ATG5 (12994), anti-ATG12 (4180), anti-Beclin-1 (3495), anti-p-PERK (Thr980, 3179), anti-p-eIF2 $\alpha$ (Ser51, 3398), anti-P65 (8242), anti-p-P65 (Ser536, 3033), anti-Lamin B1 (13435), anti-LAMP1 (9091), anti-IкB $\alpha$ (4814), anti-p-IкB $\alpha$ (Ser32, 2859), anti-mTOR (2983T), anti-p-mTOR (ser2448, 5536), anti-ULK1 (6439), anti-p-ULK1 (ser555, 5869), anti-p-ULK1 (ser757, 14202), antiAKT (2920), and anti-p-AKT (Ser473, 4060) were ordered from Cell Signaling Technology (Danvers, MA, USA); anti-TOM20 (11802-1-AP), anti-GM130 (11308-1-AP), anti-Bip (115871-AP), anti-ATF6 (24169-1-AP), anti-PERK (24390-1-AP), anti-eIF2 $\alpha$ (11170-1-AP), anti-ATF4 (10835-1-AP), anti-XBP1 (25997-1-AP), anti- $\beta$-actin (20536-1-AP), and anti-GFP (50430-2-AP) were ordered from Proteintech (Rosemont, IL, USA); anti-Calnexin (C4731) was ordered from Sigma-Aldrich (St. Louis, MO, USA); and anti-TIA-1 (sc-166247) was ordered from SCBT (Dallas, TX, USA). 


\subsection{Plasmids and Transfection}

The coding sequence of ASFV K205R was synthesized by GenScript and cloned into pcDNA3.1 (Invitrogen, Waltham, MA, USA) fused with an HA tag sequence or into pEGFP-C1 (Clontech, Mountain View, CA, USA). All plasmids were transfected with Lipofectamine 3000 (Invitrogen), according to the manufacturer's instructions.

\subsection{Cell Viability Assays}

The HeLa cells were seeded in 60-mm dishes at a density of $4 \times 10^{5}$ cells per dish and transfected with pcDNA3.1 (vector, $5 \mu \mathrm{g}$ ) and K205R-HA plasmid ( $5 \mu \mathrm{g}$ ) for 24 and $48 \mathrm{~h}$, respectively. The cell viability was determined with a CCK-8 cell counting assay kit (DingGuo, Beijing, China), according to the manufacturer's instructions.

\subsection{Immunoblotting Analysis}

The cells were seeded in $60-\mathrm{mm}$ dishes at a density of $7 \times 10^{5}$ cells per dish and transfected with indicated plasmids $(0-5 \mu \mathrm{g})$ for $24 \mathrm{~h}$. The cells were collected in RIPA buffer (Solarbio, Beijing, China) supplemented with protease and phosphatase inhibitor cocktail (MedChemExpress). The protein concentrations of the lysates were quantified with a BCA Protein Assay Kit (DingGuo). The protein samples were separated by SDS-PAGE and transferred to membranes (Millipore, Billerica, MA, USA), which were incubated in 5\% nonfat milk (Sangon) at room temperature for $1 \mathrm{~h}$ afterwards. The membranes were incubated with primary antibodies at $4{ }^{\circ} \mathrm{C}$ overnight and then incubated with horseradish-peroxidaseconjugated secondary antibodies (Jackson ImmunoResearch Laboratories, West Grove, PA, USA) for $1 \mathrm{~h}$. The immunoblotting results were visualized with Luminata Crescendo Western HRP Substrate (Millipore) on a GE AI600 imaging system. The densitometric analysis of target proteins was performed with the ImageJ software (https://imagej.nih.gov/ij/ accessed on 27 September 2021).

\subsection{Separation of the Soluble and Insoluble Fractions from Cells}

The cells were seeded in 60-mm dishes at a density of $7 \times 10^{5}$ cells per dish and transfected with indicated plasmids $(5 \mu \mathrm{g})$ for $24 \mathrm{~h}$. For separation of soluble and insoluble fractions, the cells were lysed in RIPA buffer (Solarbio) supplemented with protease and phosphatase inhibitor cocktail (MedChemExpress), and centrifuged at $12,000 \times g$ at $4{ }^{\circ} \mathrm{C}$ for $15 \mathrm{~min}$. The supernatant was considered the soluble fraction, whereas the insoluble pellet was directly mixed with $1 \times$ NuPage LDS Sample Buffer (Invitrogen) and heated at $100{ }^{\circ} \mathrm{C}$ for $30 \mathrm{~min}$. The extracted fractions were subjected to immunoblotting analysis.

\subsection{Nuclear and Cytoplasmic Extraction from Cells}

The cells were seeded in 60-mm dishes at a density of $7 \times 10^{5}$ cells per dish and transfected with indicated plasmids $(5 \mu \mathrm{g})$ for $24 \mathrm{~h}$. Nuclear and cytoplasmic extraction was performed with NEPER Nuclear and Cytoplasmic Extraction Reagents (Thermo Fisher Scientific, Waltham, MA, USA), according to the manufacturer's instructions. The extracted fractions were subjected to immunoblotting analysis.

\subsection{Separation of Cytosolic and Membrane Fractions from Cells}

The cells were seeded in 60-mm dishes at a density of $7 \times 10^{5}$ cells per dish and transfected with indicated plasmids $(5 \mu \mathrm{g})$ for $24 \mathrm{~h}$. Briefly, the cells were homogenized in $0.5 \mathrm{~mL}$ of homogenization buffer (10 mM HEPES pH 7.4, $10 \mathrm{mM} \mathrm{KCl,} 1.5 \mathrm{mM} \mathrm{MgCl}$, $5 \mathrm{mM}$ sodium EDTA, $5 \mathrm{mM}$ sodium EGTA, and $250 \mathrm{mM}$ sucrose) supplemented with protease and phosphatase inhibitors (MedChemExpress), then centrifuged at $1000 \times g$ at $4{ }^{\circ} \mathrm{C}$ for $7 \mathrm{~min}$. The pellet, containing the crude nuclear fraction, was discarded, and the supernatant was centrifuged at $12,000 \times g$ at $4{ }^{\circ} \mathrm{C}$ for $15 \mathrm{~min}$. The resulting supernatant comprised the cytosol, and the pellet containing the membrane fraction was dissolved in lysis buffer (10 mM Tris $\mathrm{HCl}$ pH 6.8, $100 \mathrm{mM} \mathrm{NaCl}$, 1\% SDS, 1 mM EDTA, and 1 mM EGTA) supplemented with protease and phosphatase inhibitors. The whole cell lysate, cytosolic, 
and membrane samples were mixed individually with $4 \times$ SDS loading buffer, boiled at $95{ }^{\circ} \mathrm{C}$ for $5 \mathrm{~min}$, and subjected to SDS-PAGE and immunoblotting analysis.

\subsection{The $q R T-P C R$}

The cells were seeded in 12-well plates at a density of $3 \times 10^{5}$ cells per dish and transfected with indicated plasmids $(2 \mu \mathrm{g})$ for $24 \mathrm{~h}$. Total RNA was isolated with TRIzol Reagent (TaKaRa, Shiga, Japan) and subjected to cDNA synthesis with a PrimeScript ${ }^{\mathrm{TM}}$ RT reagent Kit (TaKaRa). qRT-PCR was performed in triplicate with SYBR Premix Ex Taq (TaKaRa), according to the manufacturer's instructions. The data were normalized to the level of $\beta$-actin expression in each individual sample. The melting curve analysis indicated formation of a single product in all cases. The $2^{-\Delta \Delta \mathrm{Ct}}$ method was used to calculate relative expression changes. The primers used for qRT-PCR were as follows: $\beta$-actin-Fw: 5'-CTGAACCCCAAAGCCAACCGT-3', $\beta$-actin-Rv: 5' -TTCTCCTTGATGTCCCGCACG-3'; Atf4-Fw: 5'-CCCTTTACGTTCTTGCAAACTC-3', Atf4-Rv: 5'-GCTTCCTATCTCCTTCCGA GA-3'; Gadd34-Fw: 5'-AAGAGCCTGGAGAGAGGAGAG-3', Gadd34-Rv: 5'-GTCCCCAGG TTTCCAAAAGCA-3'; Chop-Fw: 5'-CTCAGG AGGAAGAGGAGGAAG-3', Chop-Rv: 5' GCTAGCTGTGCCACTTTCCTT-3'; Xbp1(s)-Fw: 5'-GAGTCCGCAGCAGGTG-3', Xbp1(s)Rv: 5'-CCGTCAGAATCCATGGGG-3'; Xbp1(t)-Fw: 5'-TCCGCAGCACTCAGACTACGT-3', Xbp1(t)-Rv: 5'-ATGCCCAAGAGGATATCAGACTC-3'; ERdj4-Fw: 5'-CAGAGAGATTGCA GAAGCATATGA-3' , ERdj4-Rv: 5'-GCTTCTTGGATCGAGTGTTTT-3'; Il-6-Fw: 5'-GCCTGA GGGCCATTCGGATA-3', Il-6-Rv: 5'-TGTGCCCAGTGGACAGGTTT-3'; Il-18-Fw: 5'-AGGG ACATCAAGCCGTGTTT-3', Il-18-Rv: 5'-CGGTCTGAGGTGCATTATCTGA-3'; and TnfaFw: 5'-CTGTAGGTTGCTCCCACCTG-3', Tnfa-Rv: 5'-CCAGTAGGGCGGTTACAGAC-3'

\subsection{Immunofluorescence Analysis}

The cells were seeded in 12-well plates with coverslips at a density of $3 \times 10^{5}$ cells per dish and transfected with indicated plasmids $(2 \mu \mathrm{g})$ for $24 \mathrm{~h}$. The cells were fixed with $4 \%$ paraformaldehyde in PBS for $30 \mathrm{~min}$ at room temperature and were then washed three times with PBS. The cells were permeabilized in PBS containing 0.1\% Triton X-100 and blocked with 10\% FBS in PBS. The primary antibodies were diluted with $10 \%$ FBS in PBS and incubated with the cells for $1 \mathrm{~h}$ at room temperature. After being washed with PBS, the cells were incubated with Alexa Fluor 568 goat anti-mouse IgG or Alexa Fluor 568 goat anti-rabbit IgG (Invitrogen) for $1 \mathrm{~h}$ at room temperature. The cells were finally washed in PBS and mounted in ProLong Diamond with DAPI (Invitrogen). Images were captured with a Zeiss LSM 800 confocal microscope.

\subsection{ELISA}

The cells were seeded in 12-well plates with coverslips at a density of $3 \times 10^{5}$ cells per dish and transfected with indicated plasmids $(2 \mu \mathrm{g})$ for $24 \mathrm{~h}$. Concentrations of porcine IL-18 were measured in the cell supernatants with ELISA kits (Advanced BioChemical, Lawrenceville, GA, USA), according to the manufacturer's instructions.

\subsection{Statistical Analysis}

All data were obtained from three independent experiments for quantitative analyses and are expressed as means \pm standard errors. All data were analyzed in Prism 7 software (GraphPad Software, Inc., San Diego, CA, USA) with two-tailed Student's $t$-tests, and $p<0.05$ was considered statistically significant.

\section{Results}

\subsection{Physical and Biochemical Parameters of ASFV K205R}

To preliminarily determine the function of ASFV K205R, we first analyzed its physical and biochemical parameters. K205R consisted of 205 amino acids comprising 31.48\% carbon, $50.33 \%$ hydrogen, $9.58 \%$ oxygen, $8.16 \%$ nitrogen, and $0.45 \%$ sulfur (Figure $1 \mathrm{~A}, \mathrm{~B}$ ). We analyzed the half-life of K205R with ProtParam (https:/ / web.expasy.org/protparam/ 
accessed on 12 November 2021). The estimated half-life of K205R in mammalian reticulocytes was $30 \mathrm{~h}$, which was longer than the approximately $20 \mathrm{~h}$ in yeast and $10 \mathrm{~h}$ in Escherichia coli (Figure 1C). The hydrophobicity and hydrophilicity of K205R computed with ProtScale (https: / / web.expasy.org/protscale/ accessed on 12 November 2021) indicated that K205R was more hydrophobic than hydrophilic (Figure 1D). We also analyzed the secondary and tertiary structure of K205R with Group-based Prediction System Version 5.0 (http:/ / gps.biocuckoo.cn/online_full.php accessed on 12 November 2021) and I-TASSER (https: / / zhanggroup.org/I-TASSER/ accessed on 12 November 2021). K205R consisted of four main $\alpha$-helices and five $\beta$-sheets (Figure 1E,F). Sixteen potential phosphorylation sites in K205R were indicated by Group-based Prediction System Version 5.0, which might be phosphorylated by MAPK, AKT EFF2L, PIKK, IKK, and PEK kinases (Figure 1E,G).

ASFV K205R amino acid sequence (205 aa):

MVEPREQFFQDLLSAVDQQMDTVKND I KD I MKEKTSFMVSFEN F I ERYDTME KN I QD LQNKYEEMAAN LMTVMTDTK I QLGA I I AQLE I LM INGT PLPAKGA I I AQLE I LM INGTPLPAKS PPASGKTSE TPKKNPTNAMFFTRSEWASSNTFREK FLTPE IQA I LDEQFANKTG I ERLHAE GLYMWRTQFSDEQKKMVKEMMKK

C

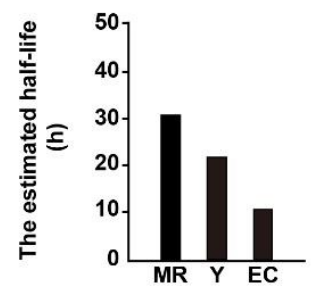

MR: mammalian reticulocytes, in vitro Y: Yeast, in vivo

EC: Escherichia coli, in vivo
B

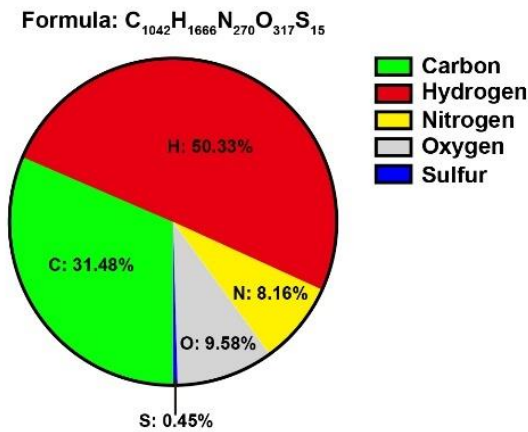

D

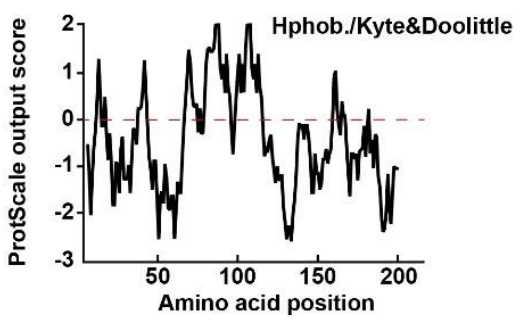
Second structure:( $/$ a-helix - coil $\Rightarrow \beta$-sheet)

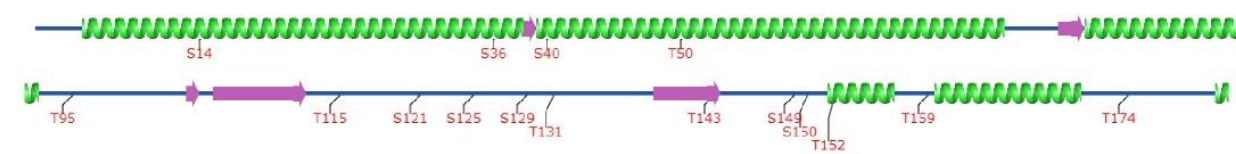

$\mathbf{F}$

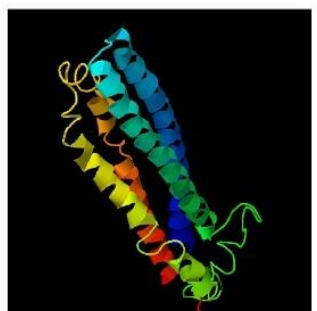

G

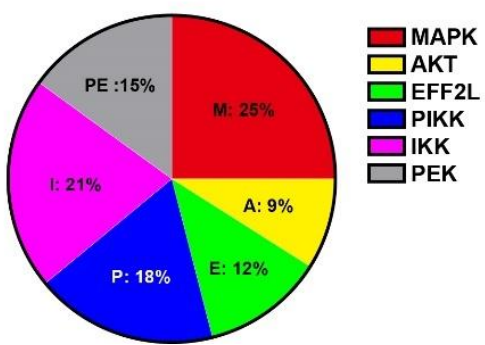

Figure 1. Physical and biochemical parameters of ASFV K205R. (A) Amino acid sequence of K205R. (B) Atomic composition of K205R. (C) Predicted half-life of K205R in mammalian reticulocytes, yeast and Escherichia coli. (D) Predicted hydropathicity and hydrophobicity of K205R. (E) Secondary structure and predicted phosphorylation sites of K205R. (F) Visualization of the predicted tertiary structure of K205R. (G) Distribution of phosphorylation sites in kinase families.

\subsection{Subcellular Localization of ASFV K205R}

To better understand K205R's function, we constructed a plasmid for the expression of K205R fused with an HA tag. We transfected the plasmid into the 3D4/21 and HeLa cells and detected its expression by immunoblotting, which confirmed the expression of 
K205R-HA in both cell lines (Figure 2A). Furthermore, CCK-8 cell counting assay showed that K205R-HA resulted in lower cell viability than that of control cells (Figure 2B), indicating that K205R might affect cell viability.

A

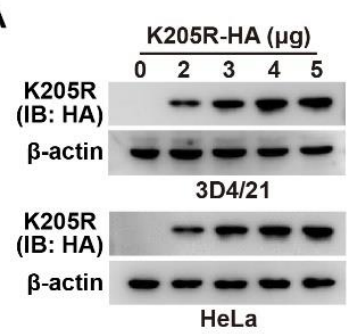

B

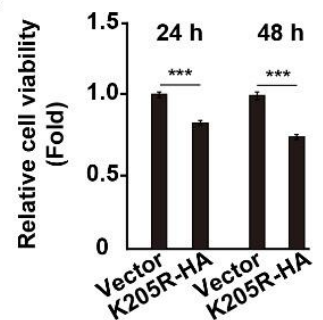

E

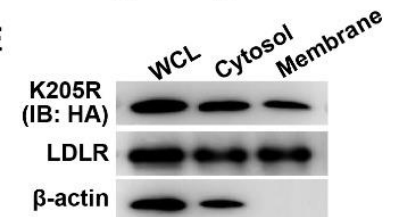

$\mathbf{F}$

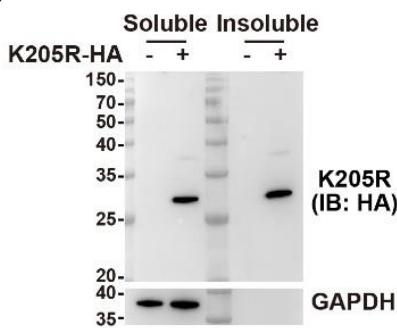

C
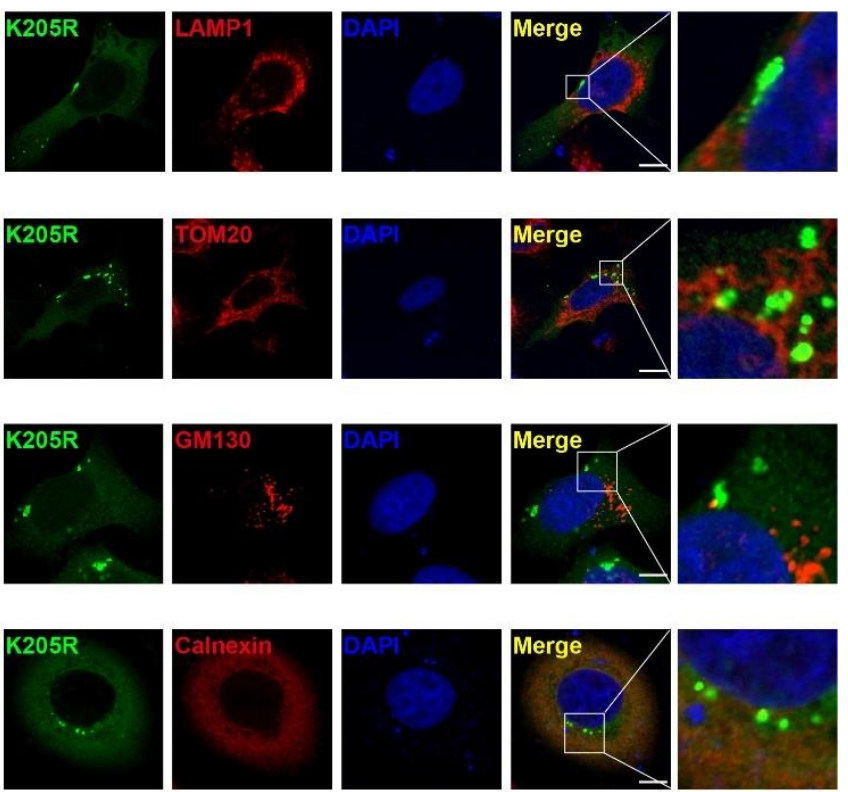

D
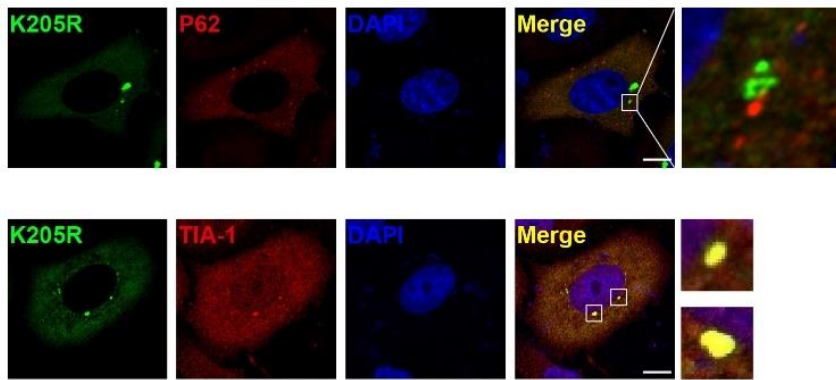

Figure 2. Expression and subcellular localization of K205R. (A) 3D4/21 and HeLa cells were transfected with K205R-HA plasmid as indicated for $24 \mathrm{~h}$. The expression of K205R-HA was detected with immunoblotting analysis. (B) HeLa cells were transfected with empty vector or K205R-HA plasmid for 24 or $48 \mathrm{~h}$. Cell viability was assessed with CCK-8 assays. ${ }^{* * *} p<0.001$. (C) HeLa cells were transfected with K205R-GFP plasmid for $24 \mathrm{~h}$. Colocalization of K205R with LAMP1 (lysosome), TOM20 (mitochondria), GM130 (Golgi), and calnexin (ER) was analyzed with immunofluorescence analysis. Scale bar: $10 \mu \mathrm{m}$. (D) HeLa cells were transfected with K205R-GFP plasmid for $24 \mathrm{~h}$. Colocalization of K205R with P62 (aggrephagy marker) and TIA-1 (SG marker) was determined with immunofluorescence analysis. Scale bar: $10 \mu \mathrm{m}$. (E) HeLa cells were transfected with K205R-HA plasmid for $24 \mathrm{~h}$. The distribution of K205R in the cytosolic and membrane fractions was detected with immunoblotting analysis. (F) HeLa cells were transfected with empty vector or K205R-HA plasmid for $24 \mathrm{~h}$. The distribution of K205R in soluble and insoluble fractions was detected with immunoblotting analysis.

We then examined the subcellular distribution of K205R through co-localization assays with markers of lysosomes (indicated by LAMP1), mitochondria (indicated by TOM20), Golgi bodies (indicated by GM130), and ER (indicated by calnexin). K205R exhibited no clear co-localization with LAMP1, TOM20, and GM130, thus suggesting that K205R scarcely localized to lysosomes, mitochondria, and Golgi bodies (Figure 2C). K205R was diffusely distributed in the cells in a degree similar to calnexin (Figure 2C). Notably, K205R was found in punctate structures, in addition to areas with diffuse distribution (Figure 2C). Protein punctate structures may form as protein aggregates or stress granules (SGs) [21,22]. Protein 
aggregates interact with the selective autophagy receptor P62 and subsequently undergo aggrephagy [21]. We did not observe co-localization of K205R with P62, indicating that K205R did not form protein aggregates (Figure 2D). Interestingly, K205R clearly colocalized with the SG marker protein TIA-1 (Figure 2D). We further performed cell fractionation analysis to identify the localization of K205R in the cytosolic and membrane fractions. The low-density lipoprotein receptor is a cell surface receptor that is recycled in the cytosol after ligand binding [23]. K205R was distributed in both the cytosolic and membrane fractions, similar to the low-density lipoprotein receptor (Figure 2E). In addition, K205R was present in soluble and insoluble forms in the cells (Figure $2 \mathrm{~F}$ ).

\subsection{ASFV K205R Activates ER Stress}

Cellular stress, such as ER stress, is a strong inducer of SG formation [24]. Therefore, we sought to determine whether K205R induces ER stress. There are three ER stress sensor pathways, IRE1, PERK and ATF6, which are critical to maintain ER homeostasis [25]. Using immunoblotting analysis, we detected that the expression of K205R in 3D4/21 cells stimulated ER stress in a K205R dose-dependent manner, as indicated by enhanced expression of ATF6 and phosphorylation of PERK, as well as the downstream effectors of ER stress, such as Bip, phosphorylated eIF2 $\alpha$, ATF4, and XBP1 (Figure 3A). This result suggested that K205R activated the IRE1, PERK, and ATF6 signaling pathways. We also obtained similar results in HeLa cells (Figure 3B). To further confirm the role of K205R in triggering ER stress, we performed qRT-PCR analysis to examine the transcript level of ER stress-responsive genes. The mRNA levels of ERdj4, processed Xbp1 mRNA [Xbp1(s)/Xbp1(t)], Atf4, Gadd34, and Chop were all up-regulated in response to K205R expression (Figure 3C-G). Treatment of cells with a PERK inhibitor (GSK2606414, GSK) abolished K205R-induced phosphorylation of PERK and eIF2 $\alpha$ (Figure 3H). Together, these results demonstrated that K205R induced ER stress.

A
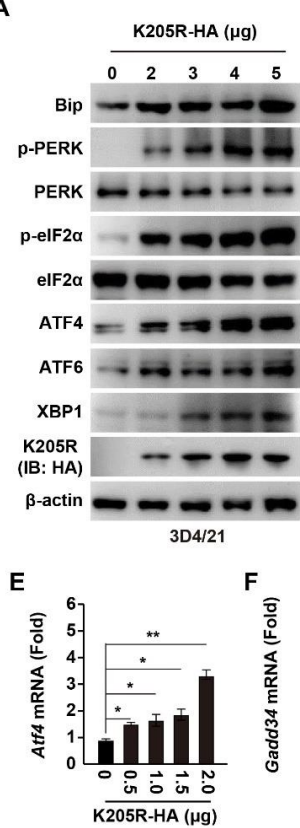

B
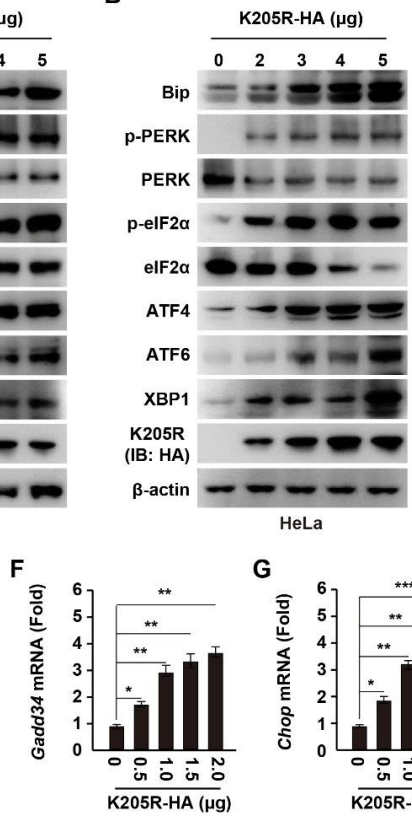

G

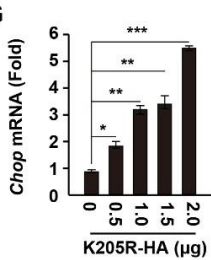

C

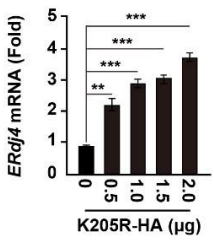

D

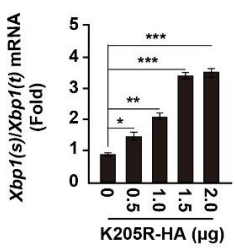

H

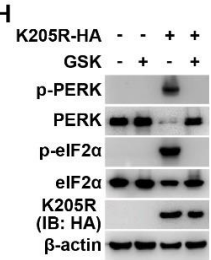

Figure 3. ASFV K205R induces ER stress. (A,B) 3D4/21 (A) and HeLa (B) cells were transfected with K205R-HA plasmid as indicated for $24 \mathrm{~h}$. Bip, p-PERK, PERK, p-eIF2 $\alpha$, eIF2 $\alpha$, ATF4, ATF6, XBP1, K205R-HA, and $\beta$-actin were assessed with immunoblotting analysis. (C-G) 3D4/21 cells were transfected with K205R-HA plasmid as indicated for $24 \mathrm{~h}$. The mRNA levels of ERdj4 (C),

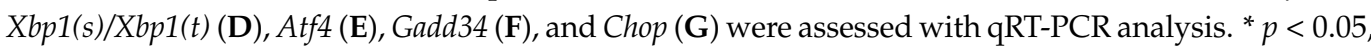
${ }^{* *} p<0.01,{ }^{* * *} p<0.001$. (H) 3D4/21 cells were transfected with K205R-HA plasmid and treated with GSK2606414 (GSK, $10 \mu \mathrm{M}$ ) as indicated for $24 \mathrm{~h}$. p-PERK, PERK, p-eIF2 $\alpha$, eIF2 $\alpha$, K205R-HA, and $\beta$-actin were assessed with immunoblotting analysis. 


\subsection{ASFV K205R Activates Autophagy}

We next attempted to examine whether K205R activates autophagy, given that ER stress is a potent trigger of autophagy [26]. We transfected K205R-GFP plasmids into the HeLa cells and then detected LC3 with immunofluorescence analysis. In the control cells, LC3 was spread throughout the cytosol and nucleus (Figure 4A). In the K205R-expressing cells, LC3 formed punctate structures, a characteristic of autophagosamal membrane formation (Figure 4A,B). We also examined K205R-induced autophagy by immunoblotting analysis. As shown in Figure 4C, increased expression of K205R resulted in enhanced expression levels of L3-II, ATG5, ATG12, and Beclin-1. When autophagy is activated, the selective autophagy receptor P62 is degraded in lysosomes and serves as an indicator of autophagic flux [21]. We observed that P62 expression decreased in response to K205R expression (Figure 4C). Bafilomycin A1, an inhibitor of the fusion of autophagosomes and lysosomes, can be used to analyze autophagic flux [27]. In the K205R expressing cells, bafilomycin A1 induced more LC3 accumulation than that in the control cells, but caused no P62 degradation (Figure 4D).

A

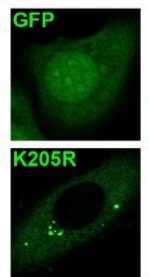

C

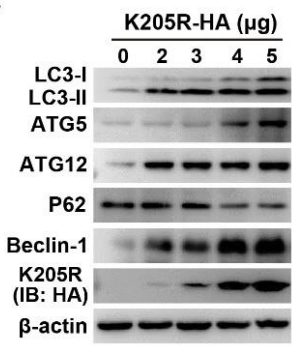

$\mathbf{F}$
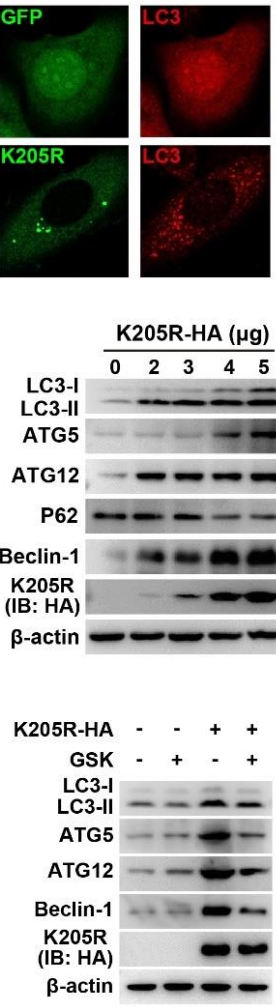

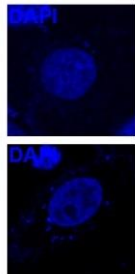

D

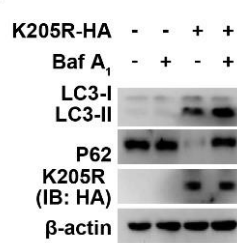

G

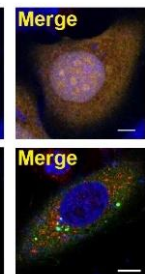

B

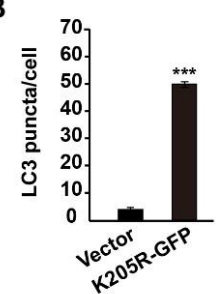

$E$

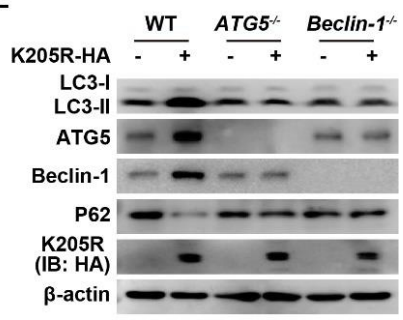

Figure 4. ASFV K205R activates autophagy. (A) HeLa cells were transfected with empty vector or K205R-GFP plasmid for $24 \mathrm{~h}$. LC3 was monitored with immunofluorescence analysis. Scale bar: $10 \mu \mathrm{m}$. (B) Quantification of LC3 puncta per cell from A $(n=30)$. ${ }^{* * *} p<0.001$. (C) 3D4/21 cells were transfected with K205R-HA plasmid as indicated for $24 \mathrm{~h}$. LC3-I, LC3-II, ATG5, ATG12, P62, Beclin-1, K205R-HA, and $\beta$-actin were assessed with immunoblotting analysis. (D) 3D4/21 cells were transfected with K205R-HA plasmid and treated with bafilomycin A1 $(10 \mu \mathrm{M})$ as indicated for $24 \mathrm{~h}$. LC3-I, LC3-II, ATG5, ATG12, P62, Beclin-1, K205R-HA, and $\beta$-actin were assessed with immunoblotting analysis. (E) PK15 WT, ATG5 ${ }^{-/-}$, and Beclin-1 $1^{-/-}$cells were transfected with K205R-HA plasmid as indicated for $24 \mathrm{~h}$. LC3-I, LC3-II, ATG5, ATG12, P62, Beclin-1, K205R-HA, and $\beta$-actin were assessed with immunoblotting analysis. (F) 3D4/21 cells were transfected with K205R-HA plasmid and treated with GSK $(10 \mu \mathrm{M})$ as indicated for $24 \mathrm{~h}$. LC3-I, LC3-II, ATG5, ATG12, Beclin-1, K205R-HA, and $\beta$-actin were assessed with immunoblotting analysis. (G) 3D4/21 cells were transfected with K205R-HA plasmid as indicated for $24 \mathrm{~h}$. AKT, p-AKT, mTOR, p-mTOR, ULK1, p-ULK1 ser555, p-ULK1 ser757, K205R-HA, and $\beta$-actin were assessed with immunoblotting analysis. 
Given that ATG5 and Beclin-1 are essential for the formation of autophagosomes [28], we verified the role of K205R in autophagy induction in $A T G 5^{-/-}$and Beclin-1-/- cells. K205R failed to induce autophagy in the $A T G 5^{-/-}$and Beclin-1 ${ }^{-/-}$cells, as indicated by immunoblotting analysis of LC3-II, ATG5, ATG12, and Beclin-1 (Figure 4E). Inhibition of PERK by GSK in the K205R-transfected cells resulted in lower expression of LC3-II, ATG5, ATG12, and Beclin-1 than that in the K205R-transfected cells, further suggesting that K205R activated autophagy through ER stress (Figure 4F). The AKT/mTOR pathway negatively regulates unc-51 like autophagy activating kinase 1 (ULK1), and hence autophagy [29]. Therefore, we attempted to determine whether the AKT/mTOR/ULK1 signaling pathway is involved in K205R-induced autophagy. We observed that phosphorylated AKT and mTOR decreased when cells expressed K205R (Figure 4G). This result indicated that the AKT/mTOR pathway was inhibited by K205R. Phosphorylation of ULK1 Ser555 was enhanced, whereas phosphorylation of ULK1 Ser757 was decreased in response to K205R expression, suggesting that K205R activated ULK1 (Figure 4G). Collectively, these data indicated that K205R activated autophagy through the AKT/mTOR/ULK1 signaling pathway.

\subsection{ASFV K205R Activates the NF- $\kappa B$ Signaling Pathway}

It is known that ER stress can elicit proinflammation [25], therefore we sought to determine whether K205R activates the NF- $\mathrm{kB}$ signaling pathway. Phosphorylation of the $\mathrm{I} \kappa \mathrm{B} \alpha$ and $\mathrm{P} 65$ subunits of nuclear factor kappa B (NF- $\mathrm{B}$ ) is essential for P65 translocation into the nucleus and subsequent NF- $\mathrm{KB}$ activation [30]. We observed that K205R expression resulted in the phosphorylation of $\mathrm{I} \kappa \mathrm{B} \alpha$ and $\mathrm{P} 65$, as indicated by immunoblotting analysis (Figure 5A). Immunofluorescence analysis suggested that P65 was translocated into the nucleus in the presence of K205R expression (Figure 5B). We further analyzed NF- $\mathrm{BB}$ activation by cell fractionation analysis of P65 with $\beta$-actin as a cytosolic marker and Lamin B1 as a nuclear marker. As shown in Figure 5C, LPS (a well-known NF- $\mathrm{kB}$ activator) stimulated P65's phosphorylation and translocation into the nucleus (Figure 5C). Expression of K205R-HA with simultaneous treatment of cells with LPS (K205R-HA + LPS) promoted the translocation of phosphorylated P65 into the nucleus (Figure 5C).

Given that NF- $\kappa B$ activation triggers the expression of proinflammatory cytokines, we next examined the transcription of $I l-6, I l-18$, and Tnfa by qRT-PCR analysis. K205R increased the mRNA levels of $I l-6, I l-18$, and Tnfa in a K205R dose-dependent manner (Figure 5D). K205R stimulated IL-18 secretion into the culture medium, as indicated by ELISA analysis (Figure 5E). We further verified K205R-induced NF- $\kappa$ B activation in 3D4/21 P65 $5^{-1-}$ cells. Neither LPS nor K205R stimulated the transcription of Il-6 and Il-18 in the

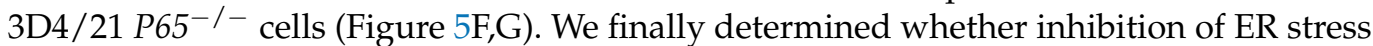
by a PERK inhibitor might abrogate K205R-mediated activation of the NF- $\kappa$ B signaling pathway. In the K205R expressing cells, GSK treatment prevented the phosphorylation of I $\mathrm{B} \alpha$ and P65 (Figure 5H). GSK treatment also inhibited the transcription of $I l-6, \mathrm{Il}-18$, and Tnfa, as well as the secretion of IL-18 when K205R-HA was expressed in cells (Figure 5I,J). Together, these results demonstrated that K205R activated the NF- $\mathrm{BB}$ signaling pathway. 
A

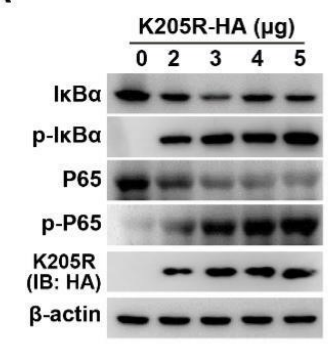

C

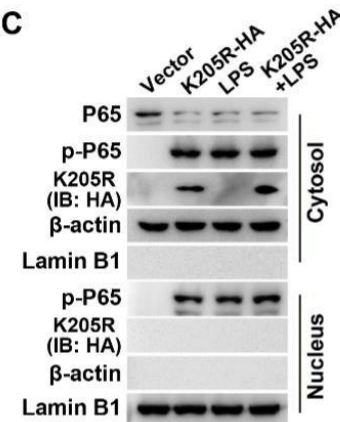

E

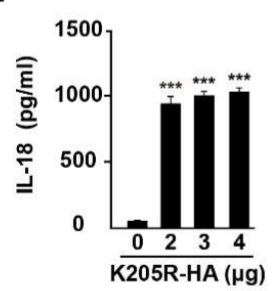

H

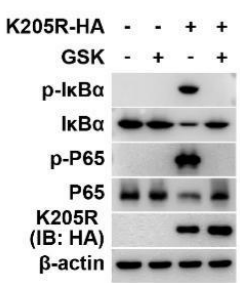

$\mathbf{F}$
B

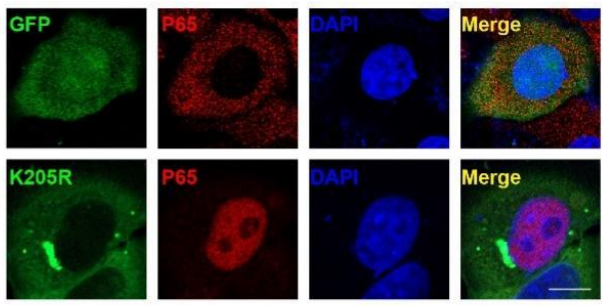

D
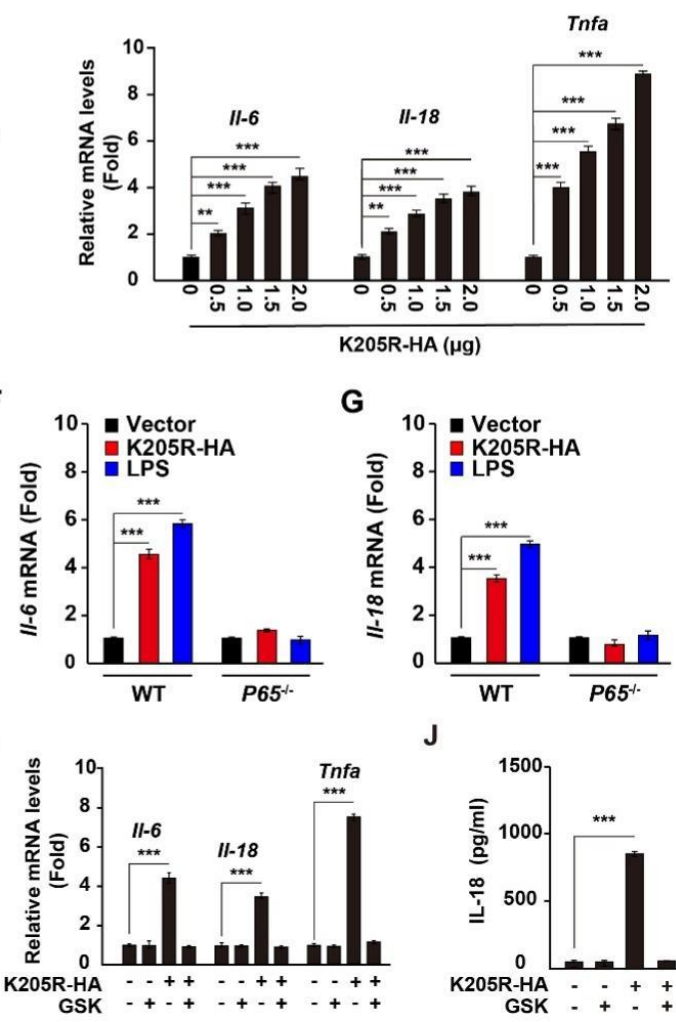

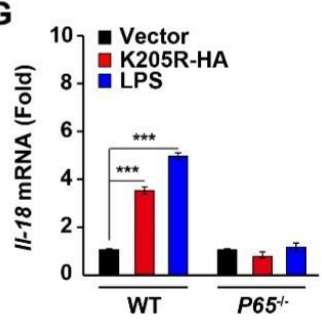

$\mathbf{J}$

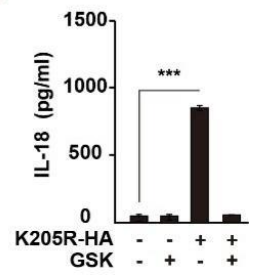

Figure 5. ASFV K205R activates the NF- $\kappa$ B signaling pathway. (A) 3D4/21 cells were transfected with K205R-HA plasmid as indicated for 24 h. IкB $\alpha, p-I \kappa B \alpha, P 65, p-P 65, K 205 R-H A$, and $\beta$-actin were assessed with immunoblotting analysis. (B) HeLa cells were transfected with empty vector or K205R-GFP plasmid for $24 \mathrm{~h}$. The translocation of P65 into the nucleus was assessed with immunofluorescence analysis. Scale bar: $10 \mu \mathrm{m}$. (C) HeLa cells were transfected with K205R-HA and treated with LPS $(1 \mathrm{mg} / \mathrm{mL})$ as indicated for $24 \mathrm{~h}$. P65 and p-P65 in the cytosol (indicated by $\beta$-actin) and nucleus (indicated by Lamin B1) were assessed with immunofluorescence analysis. (D) 3D4/21 cells were transfected with K205R-HA plasmid as indicated for $24 \mathrm{~h}$. The mRNA levels of Il-6, Il-18, and Tnfa were assessed with qRT-PCR analysis. ${ }^{* *} p<0.01,{ }^{* * *} p<0.001$. (E) 3D4/21 cells were transfected with K205R-HA plasmid as indicated for $24 \mathrm{~h}$. IL-18 in the medium was quantified with ELISA. ${ }^{* * *} p<0.001$. (F,G) 3D4/21 WT and $P 65^{-/-}$cells were transfected with K205R-HA and treated with LPS $(1 \mathrm{mg} / \mathrm{mL})$ as indicated for $24 \mathrm{~h}$. The mRNA levels of Il-6 (F) and Il-18 (G) were assessed with qRT-PCR analysis. ${ }^{* * *} p<0.001$. (H) 3D4/21 cells were transfected with K205R-HA plasmid and treated with GSK $(10 \mu \mathrm{M})$ as indicated for 24 h. p-IKB $\alpha, \mathrm{I} \kappa \mathrm{B} \alpha, \mathrm{p}-\mathrm{P} 65, \mathrm{P} 65, \mathrm{~K} 205 \mathrm{R}-\mathrm{HA}$, and $\beta$-actin were assessed with immunoblotting analysis. (I) 3D4/21 cells were transfected with K205R-HA plasmid and treated with GSK $(10 \mu \mathrm{M})$ as indicated for $24 \mathrm{~h}$. The mRNA levels of $I l-6$, Il-18, and Tnfa were assessed with qRT-PCR analysis. ${ }^{* * *} p<0.001$. (J) 3D4/21 cells were transfected with K205R-HA plasmid and treated with GSK $(10 \mu \mathrm{M})$ as indicated for $24 \mathrm{~h}$. IL-18 in the medium was quantified with ELISA. ${ }^{* * *} p<0.001$. 


\section{Discussion}

ASFV causes an acute and fatal disease affecting domestic pigs. Its genome encodes more than 160 proteins. Better understanding of the roles of ASFV proteins would be valuable in supporting vaccine development, given that no vaccine is available for the prevention and control of ASFV. In the present study, we examined the roles of ASFV K205R in ER stress, autophagy, and inflammation. K205R induced ER stress, thereby activating the UPR via the ATF6, IRE1, and PERK signaling pathways. Subsequently, K205R stimulated autophagy and NF- $\mathrm{kB}$ activation, which were prevented by inhibition of ER stress. Our results suggested that K205R induced ER stress and consequently activated autophagy and the NF- $\mathrm{KB}$ signaling pathway.

K205R has been shown to have high antigenicity and can be used for the detection of ASFV-specific antibodies [18]. Adenovirus-vectored K205R has been found to elicit robust immune responses in swine, suggesting that K205R may be an effective component of a prototype vaccine [17]. K205R is diffusely distributed throughout cells, and is found in viral factories [31]. We observed that K205R formed SG in cells. Moreover, K205R was localized in the cytosolic and membrane fractions. Although K205R contained four main $\alpha$-helices, it might not be a transmembrane protein. SG are membraneless ribonucleoprotein-based cellular compartments associated with the ER [32,33]. Therefore, we speculated that K205R might form SG tethered to the ER, thereby resulting in the observed membrane distribution of K205R.

Several lines of evidence indicate that ASFV modulated ER stress. Xia and colleagues have shown that ASFV P17 inhibited cell proliferation through ER stress and ROS-mediated cell cycle arrest [10]. Our data demonstrated that K205R decreased cell viability; therefore, K205 might promote either cell death or cell cycle arrest. Whether K205R induces ROS requires further investigation. ASFV induces the ATF6 branch of the UPR, but not the PERK pathways, which promote ASFV infection [11]. In contrast, ASFV does not activate ATF6-regulated CHOP and instead inhibits the induction of CHOP/GADD153 via several exogenous stimuli [13]. Our data indicated that K205R activates the IRE1, PERK, and ATF6 signaling pathways. Other ASFV proteins might possibly modulate ER stress. The role of ASFV in modulating ER stress depends on the synergistic effects of ASFV proteins that participate in ER stress. We acknowledge that more data is needed to confirm that K205R induces ER stress under physiological conditions.

Innate immunity is the front-line defense against viral infections [34]. Cyclic guanosine monophosphate/adenosine monophosphate synthase (cGAS) and STING are crucial innate immune proteins involved in cytosolic DNA sensing [35]. Although ASFV controls interferon beta production through the cGAS-STING pathway, this virus exploits autophagy to interfere with this pathway [36]. ASFV MGF-505-7R negatively regulates the cGAS-STING-mediated signaling pathway through autophagy-mediated degradation of STING [6]. Moreover, ASFV MGF505-11R promotes STING degradation by autophagy for negative regulation of the cGAS-STING signaling pathway [7]. ASFV E199L promotes cell autophagy through the interaction of PYCR2 [15]. We found that K205R activated autophagy through ER stress. The roles of ASFV E199L-induced and K205R-induced autophagy in STING degradation and innate immune evasion are worthy of further study. Notably, we found that K205R activated the NF- $\mathrm{KB}$ signaling pathway, thereby protecting the host against ASFV infection. This understanding of the complicated roles of K205R in cellular responses provides new insight into virus-host interactions.

Author Contributions: Conceptualization, B.C. and G.Z.; methodology, Q.W. and L.Z.; software, J.W. and D.S.; validation, Y.D. and D.L.; formal analysis, G.Y.; investigation, Q.W. and L.Z.; data curation, J.W.; writing - original draft preparation, B.C.; writing-review and editing, B.C.; supervision, B.C. and G.Z.; funding acquisition, B.C. and G.Z. All authors have read and agreed to the published version of the manuscript. 
Funding: This work was supported by grants of the National Natural Science Foundation of China (31941001), the Ten Thousand Talents Program for Young Talents (W03070106), and the Key Scientific Research Projects of Colleges and Universities of Henan Province (21A230010).

Institutional Review Board Statement: Not applicable.

Informed Consent Statement: Not applicable.

Data Availability Statement: All available data are presented in the article.

Conflicts of Interest: The authors declare that they have no conflict of interest.

\section{References}

1. Gao, L.; Sun, X.; Yang, H.; Xu, Q.; Li, J.; Kang, J.; Liu, P.; Zhang, Y.; Wang, Y.; Huang, B. Epidemic situation and control measures of African Swine Fever Outbreaks in China 2018-2020. Transbound. Emerg. Dis. 2021, 68, 2676-2686. [CrossRef] [PubMed]

2. Zhang, X.; Rong, X.; Li, J.; Fan, M.; Wang, Y.; Sun, X.; Huang, B.; Zhu, H. Modeling the outbreak and control of African swine fever virus in large-scale pig farms. J. Theor. Biol. 2021, 526, 110798. [CrossRef] [PubMed]

3. Mai, N.; Vu, X.; Nguyen, T.; Nguyen, V.; Trinh, T.; Kim, Y.; Kim, H.; Cho, K.; Nguyen, T.; Bui, T.; et al. Molecular profile of African swine fever virus (ASFV) circulating in Vietnam during 2019-2020 outbreaks. Arch. Virol. 2021, 166, 885-890. [CrossRef] [PubMed]

4. Correia, S.; Ventura, S.; Parkhouse, R. Identification and utility of innate immune system evasion mechanisms of ASFV. Virus Res. 2013, 173, 87-100. [CrossRef] [PubMed]

5. Li, T.; Zhao, G.; Zhang, T.; Zhang, Z.; Chen, X.; Song, J.; Wang, X.; Li, J.; Huang, L.; Wen, L.; et al. African Swine Fever Virus pE199L Induces Mitochondrial-Dependent Apoptosis. Viruses 2021, 13, 2240. [CrossRef] [PubMed]

6. $\quad$ Li, D.; Yang, W.; Li, L.; Li, P.; Ma, Z.; Zhang, J.; Qi, X.; Ren, J.; Ru, Y.; Niu, Q.; et al. African Swine Fever Virus MGF-505-7R Negatively Regulates cGAS-STING-Mediated Signaling Pathway. J. Immunol. 2021, 206, 1844-1857. [CrossRef] [PubMed]

7. Yang, K.; Huang, Q.; Wang, R.; Zeng, Y.; Cheng, M.; Xue, Y.; Shi, C.; Ye, L.; Yang, W.; Jiang, Y.; et al. African swine fever virus MGF505-11R inhibits type I interferon production by negatively regulating the cGAS-STING-mediated signaling pathway. Vet. Microbiol. 2021, 263, 109265. [CrossRef]

8. Banerjee, A.; Czinn, S.J.; Reiter, R.J.; Blanchard, T.G. Crosstalk between endoplasmic reticulum stress and anti-viral activities: A novel therapeutic target for COVID-19. Life Sci. 2020, 255, 117842. [CrossRef]

9. Cybulsky, A. Endoplasmic reticulum stress, the unfolded protein response and autophagy in kidney diseases. Nat. Rev. Nephrol. 2017, 13, 681-696. [CrossRef]

10. Xia, N.; Wang, H.; Liu, X.; Shao, Q.; Ao, D.; Xu, Y.; Jiang, S.; Luo, J.; Zhang, J.; Chen, N.; et al. African Swine Fever Virus Structural Protein p17 Inhibits Cell Proliferation through ER Stress-ROS Mediated Cell Cycle Arrest. Viruses 2020, 13, 21. [CrossRef]

11. Galindo, I.; Hernaez, B.; Munoz-Moreno, R.; Cuesta-Geijo, M.A.; Dalmau-Mena, I.; Alonso, C. The ATF6 branch of unfolded protein response and apoptosis are activated to promote African swine fever virus infection. Cell Death Dis. 2012, 3, e341. [CrossRef]

12. Nishitoh, H. CHOP is a multifunctional transcription factor in the ER stress response. J. Biochem. 2012, 151, 217-219. [CrossRef]

13. Netherton, C.L.; Parsley, J.C.; Wileman, T. African swine fever virus inhibits induction of the stress-induced proapoptotic transcription factor CHOP/GADD153. J. Virol. 2004, 78, 10825-10828. [CrossRef] [PubMed]

14. Ravanan, P.; Srikumar, I.F.; Talwar, P. Autophagy: The spotlight for cellular stress responses. Life Sci. 2017, 188, 53-67. [CrossRef] [PubMed]

15. Chen, S.; Zhang, X.; Nie, Y.; Li, H.; Chen, W.; Lin, W.; Chen, F.; Xie, Q. African Swine Fever Virus Protein E199L Promotes Cell Autophagy through the Interaction of PYCR2. Virol. Sin. 2021, 36, 196-206. [CrossRef]

16. Hernaez, B.; Cabezas, M.; Munoz-Moreno, R.; Galindo, I.; Cuesta-Geijo, M.A.; Alonso, C. A179L, a new viral Bcl2 homolog targeting Beclin 1 autophagy related protein. Curr. Mol. Med. 2013, 13, 305-316. [CrossRef] [PubMed]

17. Lokhandwala, S.; Waghela, S.D.; Bray, J.; Sangewar, N.; Charendoff, C.; Martin, C.L.; Hassan, W.S.; Koynarski, T.; Gabbert, L.; Burrage, T.G.; et al. Adenovirus-vectored novel African Swine Fever Virus antigens elicit robust immune responses in swine. PLoS ONE 2017, 12, e0177007. [CrossRef] [PubMed]

18. Wu, X.; Xiao, L.; Peng, B.; Wang, Y.; Yang, Z.; Yao, X.; Hu, L.; Lin, X. Prokaryotic expression, purification and antigenicity analysis of African swine fever virus pK205R protein. Pol. J. Vet. Sci 2016, 19, 41-48. [CrossRef] [PubMed]

19. Ming, S.L.; Zhang, S.; Wang, Q.; Zeng, L.; Zhou, L.Y.; Wang, M.D.; Ma, Y.X.; Han, L.Q.; Zhong, K.; Zhu, H.S.; et al. Inhibition of USP14 influences alphaherpesvirus proliferation by degrading viral VP16 protein via ER stress-triggered selective autophagy. Autophagy 2021, 1-21. [CrossRef]

20. Ming, S.L.; Zeng, L.; Guo, Y.K.; Zhang, S.; Li, G.L.; Ma, Y.X.; Zhai, Y.Y.; Chang, W.R.; Yang, L.; Wang, J.; et al. The Human-Specific STING Agonist G10 Activates Type I Interferon and the NLRP3 Inflammasome in Porcine Cells. Front. Immunol. 2020, 11, 575818. [CrossRef]

21. Lamark, T.; Svenning, S.; Johansen, T. Regulation of selective autophagy: The p62/SQSTM1 paradigm. Essays Biochem. 2017, 61, 609-624. [CrossRef] [PubMed]

22. Protter, D.S.W.; Parker, R. Principles and Properties of Stress Granules. Trends Cell Biol. 2016, 26, 668-679. [CrossRef] [PubMed] 
23. Gent, J.; Braakman, I. Low-density lipoprotein receptor structure and folding. Cell Mol. Life Sci. 2004, 61, 2461-2470. [CrossRef] [PubMed]

24. van Leeuwen, W.; Rabouille, C. Cellular stress leads to the formation of membraneless stress assemblies in eukaryotic cells. Traffic 2019, 20, 623-638. [CrossRef]

25. Di Conza, G.; Ho, P.C. ER Stress Responses: An Emerging Modulator for Innate Immunity. Cells 2020, 9, 695. [CrossRef]

26. Lee, W.S.; Yoo, W.H.; Chae, H.J. ER Stress and Autophagy. Curr. Mol. Med. 2015, 15, 735-745. [CrossRef]

27. Yamamoto, A.; Tagawa, Y.; Yoshimori, T.; Moriyama, Y.; Masaki, R.; Tashiro, Y. Bafilomycin A1 prevents maturation of autophagic vacuoles by inhibiting fusion between autophagosomes and lysosomes in rat hepatoma cell line, H-4-II-E cells. Cell Struct. Funct. 1998, 23, 33-42. [CrossRef]

28. Xie, Z.; Klionsky, D.J. Autophagosome formation: Core machinery and adaptations. Nat. Cell Biol. 2007, 9, 1102-1109. [CrossRef]

29. Zachari, M.; Ganley, I.G. The mammalian ULK1 complex and autophagy initiation. Essays Biochem. 2017, 61, 585-596. [CrossRef]

30. Mulero, M.C.; Huxford, T.; Ghosh, G. NF-kappaB, IkappaB, and IKK: Integral Components of Immune System Signaling. Adv. Exp. Med. Biol. 2019, 1172, 207-226. [CrossRef]

31. Gutierrez-Castaneda, B.; Reis, A.L.; Corteyn, A.; Parkhouse, R.M.; Kollnberger, S. Expression, cellular localization and antibody responses of the African swine fever virus genes B602L and K205R. Arch. Virol. 2008, 153, 2303-2306. [CrossRef] [PubMed]

32. Riggs, C.L.; Kedersha, N.; Ivanov, P.; Anderson, P. Mammalian stress granules and P bodies at a glance. J. Cell Sci. 2020, 133 , jcs242487. [CrossRef] [PubMed]

33. Lee, J.E.; Cathey, P.I.; Wu, H.; Parker, R.; Voeltz, G.K. Endoplasmic reticulum contact sites regulate the dynamics of membraneless organelles. Science 2020, 367, eaay7108. [CrossRef] [PubMed]

34. Carty, M.; Guy, C.; Bowie, A.G. Detection of Viral Infections by Innate Immunity. Biochem. Pharm. 2021, 183, 114316. [CrossRef] [PubMed]

35. Hopfner, K.P.; Hornung, V. Molecular mechanisms and cellular functions of cGAS-STING signalling. Nat. Rev. Mol. Cell Biol. 2020, 21, 501-521. [CrossRef] [PubMed]

36. Garcia-Belmonte, R.; Perez-Nunez, D.; Pittau, M.; Richt, J.A.; Revilla, Y. African Swine Fever Virus Armenia/07 Virulent Strain Controls Interferon Beta Production through the cGAS-STING Pathway. J. Virol. 2019, 93, e02298-18. [CrossRef] 